RASĀYAN J. Chem.

Vol. 13 | No. 4 |2193-2199| October - December | 2020 ISSN: 0974-1496 | e-ISSN: 0976-0083 | CODEN: RJCABP

RJC http://www.rasayanjournal.com http://www.rasayanjournal.co.in

\title{
MECHANICAL AND THERMAL PROPERTIES OF STRAINED METAL NANOPARTICLES PREPARED BY BALL MILLING METHOD
}

\author{
Purushotham Endla \\ Department of Physics, School of Sciences, SR University, Ananthasagar (V), Hasanparthy (M), \\ Warangal Urban-506371, Telangana, India. \\ E-mail: psm45456@gmail.com
}

\begin{abstract}
Mechanical properties of crystallite size and strains in tungsten (W) powders created by processing have been examined by X-ray powder diffraction. The mechanical properties of strain $(\varepsilon)$,crystallite size and the metal thermal properties of Debye-Waller factor (B), Debye temperature $\left(\theta_{\mathrm{M}}\right)$, Mean square amplitude of vibration $(<\mathrm{u}>$ ) are resolved from the half-widths and incorporated intensities of the Bragg reflections and contrasted and Scanning Electron Microscopic analysis/examination. In tungsten metal, the Debye-Waller factor is found to increment with the cross-section strain. From the relationship between's the strain and successful Debye-Waller factor, the DebyeWaller factors for zero strain have been assessed for tungsten metal. In the current work, the Hall-Williamson technique for molecule size or crystallite size estimation of high vitality processed tungsten nanopowder. The variety of vitality of opening arrangement as a component of cross-section strain has been examined.

Keywords: X-ray Diffraction, SEM, Lattice Strain, Crystallite Size, Debye-Waller Factor, Vacancy Formation Energy.
\end{abstract}

(C) RASĀYAN. All rights reserved

\section{INTRODUCTION}

Tungsten has several unique properties that establish it as a candidate material for fusion reactor applications. These properties include high melting point, high thermal conductivity, excellent mechanical properties at elevated temperatures, low tritium retention, low sputtering yield. However, tungsten also has several serious deficiencies related to brittleness of the material, including room-temperature brittleness, crystallization brittleness, and irradiation induced brittleness, which present risks in fusion reactor applications. The brittleness of tungsten may lead to poor thermal shock resistance when exposed to extremely high cyclic heating.

Influenceof tool revolving on mechanical properties of some metals and alloy had been developed as next generation engineering production ${ }^{1,2}$ and with some thermal and mechanical properties and engineering applications. ${ }^{3,4}$ Physical and concoction properties of these materials are profoundly size ward. Thusly, it is imperative to create novel methods for the blend of nanomaterials. TEM is one of the amazing procedures for crystallite size estimation, it has certain restrictions. Since TEM pictures speak to just a nearby district, numerous examples and pictures are required to give a normal data to the whole example. Not just this, the TEM test readiness technique is an included and tedious one. The XRD procedure is liberated from these restrictions. X-beam diffraction is, then again, a straightforward and simpler methodology for assurance of crystallite size of powder tests.

The Debye-Waller factor is an important lattice dynamical property. There is considerable X-ray work on the Debye-Waller factors of Tungsten metal ${ }^{5}$ and $\mathrm{CuInSe}_{2}{ }^{6}$. But it is interesting to study the effect of lattice strains on the Debye-Waller factors of this metal. The X-Ray study and effect of lattice strain on the Debye-Waller factors of some metals $\mathrm{Ag}^{7}, \mathrm{Pt}^{8}, \mathrm{Ni}^{9}$. In this process, the strains produced during grinding have a significant effect on the Debye-Waller factors measured from X-ray diffraction intensities.

Sirdeshmukh et $a l^{10}$ observed the effect of lattice strains on the Debye-Waller factors in semiconductor powder materials. Gopi Krishna and Sirdeshmukh ${ }^{11}$ studied the effect of lattice strains on the Debye-

Rasayan J. Chem., 13(4), 2193-2199(2020)

http://dx.doi.org/10.31788/ RJC.2020.1345788

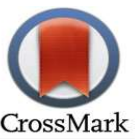


RASĀYAN J. Chem.

Vol. 13 | No. 4 |2193-2199| October - December | 2020

Waller factor of ytterbium metal. In the present investigation, the results of a systematic study of the effect of lattice strain on the Debye-Waller factors and crystallite size of face-centered cubic W metal is reported. These results are being reported for the first time.

\section{EXPERIMENTAL}

The powder samples were obtained by gently filing highly pure $\mathrm{W}$ metal ingots with a jeweller's file. A part of this powder was used to prepare the initial sample. The remaining powder was subjected to milling in a ball mill for $5,10,15,20,25,30,35$ and 40 hours to produce strains. X-ray diffractograms were recorded with the initial sample and with samples prepared after each spell of milling. The diffractograms were obtained with a Philips CWU 3710 X-ray powder diffractometer in the $2 \theta$ range $20-120^{\circ}$ using filtered $\mathrm{CuK}_{\alpha}$ at a goniometer speed of $0.5^{\circ} \mathrm{per}$ minute and a chart speed of $20 \mathrm{~mm} / \mathrm{min}$. The XRD patterns of $\mathrm{W}$ nanopowder is given in Fig.-1.

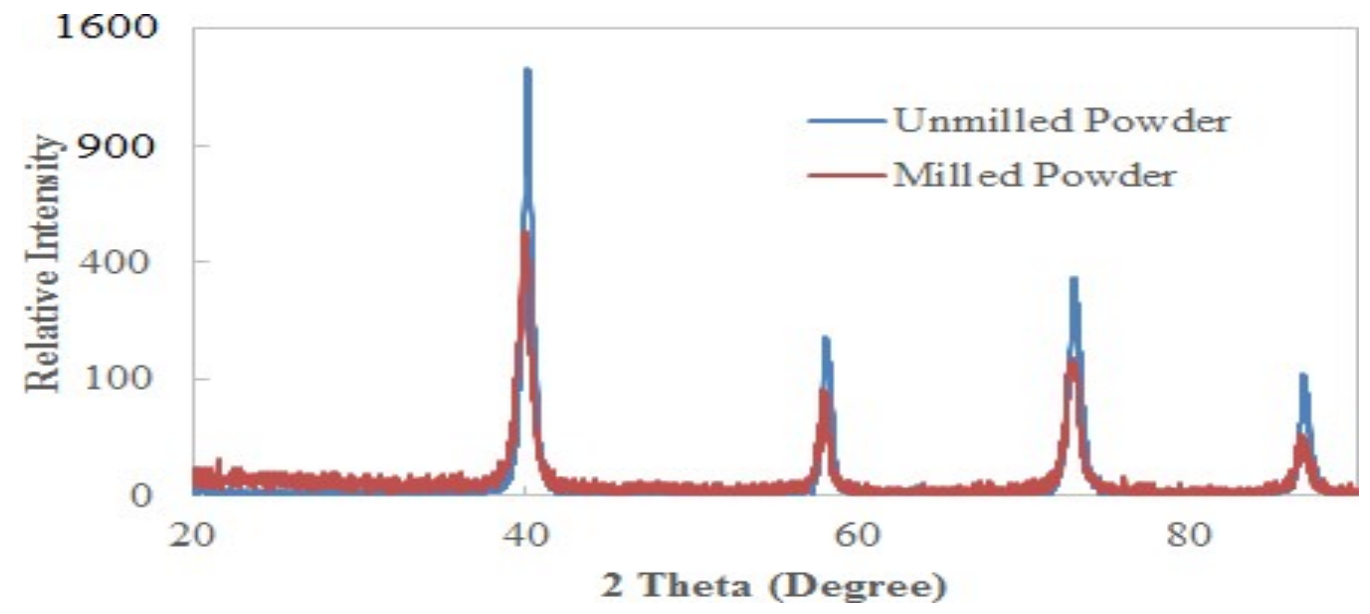

Fig.-1: XRD Pattern Comparing Unmilled Powder (For 0 hours) and Milled Powder (for 40 hours) W Powders

Figure-1 shows a comparison of the XRD pattern of un-milled and milled nano-W powders. All measurements were made at room temperature. The observed integrated intensities have been corrected for thermal diffuse scattering using the method of Chipman and Paskin ${ }^{12}$. For the relative intensity method, the expression for the observed intensities $\mathrm{I}_{0}$ is given by-

$$
\mathrm{I}_{0}=\mathrm{CL}_{\mathrm{p}} \mathrm{JF}_{\mathrm{T}}^{2}
$$

Where, $\mathrm{L}_{\mathrm{p}}$ is the Lorentz-polarization factor, $\mathrm{J}$, the multiplicity factor, $\mathrm{F}_{\mathrm{T}}$ the structure factor and $\mathrm{C}$ is a constant. For a flat powder specimen, the absorption correction is independent of the angle $\theta$, Klug and Alexander ${ }^{13}$ and, hence, is lumped with the constant. The structure factor $F_{T}$ in terms of the structure factor $\mathrm{F}$ for the static lattice is given by-

$$
\mathrm{F}_{\mathrm{T}}=\mathrm{Fe}^{-\mathrm{M}}
$$

We may also write Eq. (1) as:

$$
\mathrm{I}_{0}=I_{c} e^{-2 B\left(\frac{\sin \theta}{\lambda}\right)^{2}}
$$

where $\mathrm{I}_{\mathrm{c}}$ is the intensity corresponding to the static lattice and is given by-

$$
\mathrm{I}_{\mathrm{c}}=\mathrm{L}_{\mathrm{p}} \mathrm{JF}^{2}
$$

For crystals with fcc structure, the structure factor $\mathrm{F}$ is given by-

$$
\mathrm{F}=4 \mathrm{f}
$$


RASĀYAN J. Chem.

Vol. 13 | No. 4 |2193-2199| October - December | 2020

Where, $\mathrm{f}$ is the atomic scattering factor. Values of the atomic scattering factor were taken from Cromer and Waber ${ }^{14}$ and International Tables for X-ray Crystallography ${ }^{15}$, and have been corrected for dispersion, Cromer and Liberman. ${ }^{16}$

From Eq. (3) it can be seen that $\log \left(I_{0} / I_{c}\right)$ is linearly related to $(\sin \theta / \lambda)^{2}$. By the least square treatment of data, B was determined. From the Debye-Waller theory,

$$
\mathrm{B}=\left(\frac{8 \pi^{2}}{3}\right)<\mathrm{u}^{2}>
$$

for a cubic crystal, where $\left\langle\mathrm{u}^{2}>\right.$ is the mean-square amplitude of vibration. Further, B, may also be expressed as

$$
\mathrm{B}=\left(\frac{6 h^{2}}{m k_{B} T}\right) \mathrm{W}(\mathrm{x})
$$

Where $\mathrm{m}$ is the mass, $\mathrm{T}$ the absolute temperature and $\mathrm{h}$ and $\mathrm{k}_{\mathrm{B}}$ are the Planck and the Boltzmann constants respectively. The function $\mathrm{W}(\mathrm{x})$ is given by-

$$
\mathrm{W}(\mathrm{x})=\left[\frac{\phi(x)}{x^{2}}+\frac{1}{4 x}\right]
$$

Where $\varnothing(x)$ is the Debye function and $x=\theta_{M} / T, \theta_{M}$ being the Debye temperature. Benson and Gill $^{17}$ have tabulated values of $\mathrm{W}(\mathrm{x})$ for a wide range of $\mathrm{x}$ for small increments, from which $\theta_{\mathrm{M}}$ can be obtained from the value of $B$.

\section{Lattice Strain and Particle Size Determination}

At the point when the size of the individual precious stones is not exactly about $100 \mathrm{~nm}$ the expression "molecule size" is typically utilized. At the point when the crystallites of material are littler than $100 \mathrm{~nm}$, they have excessively little various equal diffraction planes thus they produce expanded diffraction tops rather than a sharp pinnacle. Cross-section strain present in the example is another reason for the widening of Bragg diffraction tops. Likewise, there are instrumental factors, for example, uncertain $\alpha 1$ and $\alpha 2$ tops, flawed centering which leads to the line expanding. There are different strategies by and by to evaluate the molecule size. X-beam diffraction is a more straightforward and simpler methodology for the assurance of exact crystallite size and the cross-section strain in powder tests. The guideline engaged with the X-beam diffraction approach is the exact measurement of the widening of the Bragg diffraction tops. Scherrer condition, Hall-Williamson strategy and Warren-Averbach strategy are a portion of the strategies dependent on this rule. Of the above techniques, the Scherrer condition strategy for the estimation of molecule size doesn't consider expanding because of the cross-section strain present in the example. As such in the current examination, the cross-section strains have been evaluated utilizing the Hall-Williamson strategy. As of late, Bharati et al18 have utilized this technique to appraise the crosssection strain and molecule sizes of silver nanoparticles and composite silver nanoparticles. In this strategy, the vital expansiveness of the diffraction top is resolved. The indispensable broadness is given by the incorporated force separated by the greatest power. In this manner, the watched top widening Bo might be spoken to as:

$$
\mathrm{B}_{\mathrm{o}}=\mathrm{B}_{\mathrm{i}}+\mathrm{B}_{\mathrm{r}}
$$

Where $B_{0}$ is the observed peak broadening in radians, $B_{i}$ is the instrumental broadening in radians and $B_{r}$ is the broadening due to the small particle size and lattice strain. The instrumental widening/ broadening has been evaluated utilizing an unadulterated without strain fine sodium chloride powder test exposed to XRD under indistinguishable conditions as those for the stressed metallic powders. Equation- 9 holds great if the diffraction tops show Cauchy profile. Notwithstanding, when the diffraction tops are somewhat Cauchy and incompletely Gaussian for profiles, the accompanying connection between $\mathrm{Bo}, \mathrm{Bi}$ and $\mathrm{Br}$ hold great, Bharati et al. ${ }^{18}$

$\mathrm{B}_{\mathrm{r}}=\left[\left(\mathrm{B}_{\mathrm{o}}-\mathrm{B}_{\mathrm{i}}\right)\left(\mathrm{B}_{\mathrm{o}}{ }^{2}-\mathrm{B}_{\mathrm{i}}^{2}\right)^{1 / 2}\right]^{1 / 2}$

Now, according to Scherrer equation, the broadening due to small particle size may be expressed as: 
RASĀYAN J. Chem.

Vol. 13 | No. 4 |2193-2199| October - December | 2020

$\mathrm{B}_{\mathrm{c}}=\frac{\mathrm{k} \lambda}{\mathrm{t}} \cos \theta$

Where $B_{c}$ is the broadening solely due to small crystallite size, $\mathrm{K}$ a constant whose value depends on particle shape and usually taken as unity, $\mathrm{t}$ the crystallite size in nanometers, $\theta$ the Bragg angle and $\lambda$ is the wavelength of the incident X-ray beam in nanometers.Similarly, according to Wilson ${ }^{19}$, the broadening due to lattice strain may be expressed by the relation,

$\mathrm{B}_{\mathrm{s}}=\varepsilon \tan \theta$

Where $B_{s}$ is the peak broadening due to lattice strain and $\varepsilon$ the strain distribution within the material and $\theta$ is the Bragg angle. Based on Eqs. (11) and (12) the total peak broadening $\mathrm{B}_{\mathrm{r}}$ may be expressed as,

$$
\mathrm{B}_{\mathrm{r}}=\frac{\mathrm{k} \lambda}{\mathrm{t} \cos \theta}+\varepsilon \tan \theta
$$

Which can be written as:

$$
\mathrm{B}_{\mathrm{r}} \cos \theta=\frac{\mathrm{k} \lambda}{\mathrm{t}}+\varepsilon \sin \theta
$$

The plot of $B_{r} \cos \theta / \lambda$ versus $\sin \theta / \lambda$ is a straight line with slope equal to $\varepsilon$ and hence the particle size ' $t$ ' can be estimated from the intercept. Typical Hall-Williamson plot between $B_{\mathrm{r}} \cos \theta / \lambda$ and $\sin \theta / \lambda$ is $\operatorname{shown}$ in Fig.-2.

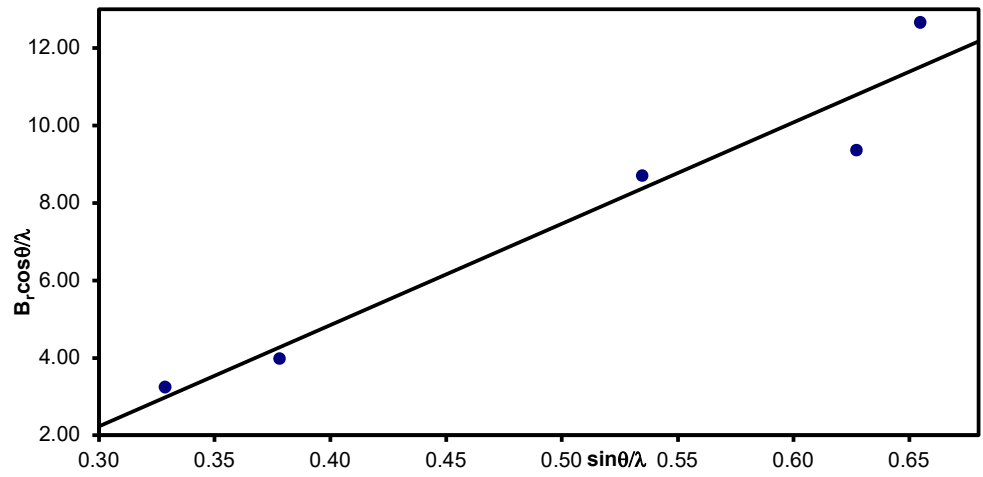

Fig.-2: Plot of $\mathrm{B}_{\mathrm{r}} \cos \theta / \lambda$ Vs $\sin \theta / \lambda$ for $\mathrm{W}$ after milling for 25 hours

The lattice strains were determined from the plot of $B_{r} \cos \theta / \lambda$ against $\sin \theta / \lambda$ following standard procedures $^{20}$. The measured half-widths were corrected for instrumental broadening concerning a pure strain-free silicon powder. The variation of particle size with milling time is within the limits of experimental errors. This shows that while the milling is enough to create strains, it affects the particle size to a measurable extent. A typical Hall-Williamson plot is shown in Figure 2 for Ag after grinding for 25 hours.

\section{RESULTS AND DISCUSSION}

The values of the lattice strain, crystallite size, root mean square amplitude of vibrations, Debye-Waller factor and Debye temperature of W powders, ground for different durations, obtained in the present study are given in Table-1. As the objective of the present work is to investigate the strain dependence of Debye-Waller factors, the variation of the lattice strain $(\varepsilon)$ and Debye-Waller factor (B) for different milling times for fcc metal $\mathrm{W}$ is shown in Fig.-3.

Table-1: Values of Lattice Strain ( $\varepsilon$ ), Crystallite Size (t), Debye-Waller Factor (B), Root Mean Square Amplitudes of Vibration $<\mathrm{u}>$, Debye Temperature $\left(\theta_{\mathrm{M}}\right)$ and Energy of Vacancy Formation $\left(\mathrm{E}_{\mathrm{f}}\right)$ of Strained Tungsten

Nanopowders

\begin{tabular}{c|c|c|c|c|c|c|c}
\hline Metal & Milling time & $\varepsilon \times 10^{3}$ & $\mathrm{t}(\mathrm{nm})$ & $<\mathrm{u}>(\AA)$ & $\mathrm{B}\left(\AA^{2}\right)$ & $\theta_{\mathrm{M}}(\mathrm{K})$ & $\mathrm{E}_{\mathrm{f}}(\mathrm{eV})$ \\
\hline $\mathrm{W}$ & 0 & 1.23 & 500 & $0.0804(3)$ & $0.17(2)$ & $336(3)$ & 7.17 \\
\hline & 5 & 1.38 & 118 & $0.0830(2)$ & $0.18(1)$ & $325(5)$ & 6.71 \\
\hline & 10 & 1.47 & 91 & $0.0850(3)$ & $0.19(1)$ & $318(3)$ & 6.42 \\
\hline
\end{tabular}


RASĀYAN J. Chem.

Vol. 13 | No. 4 |2193-2199| October - December | 2020

\begin{tabular}{l|l|l|l|l|l|l|l}
\hline & 15 & 1.67 & 88 & $0.0893(4)$ & $0.21(2)$ & $303(13)$ & 5.83 \\
\hline & 20 & 1.99 & 69 & $0.0935(6)$ & $0.21(2)$ & $288(9)$ & 5.27 \\
\hline & 25 & 2.08 & 46 & $0.1031(6)$ & $0.28(1)$ & $261(6)$ & 4.33 \\
\hline & 30 & 2.18 & 38 & $0.1085(4)$ & $0.31(3)$ & $248(23)$ & 3.91 \\
\hline & 35 & 3.66 & 29 & $0.1217(6)$ & $0.39(4)$ & $220(31)$ & 3.07 \\
\hline & 40 & 4.77 & 21 & $0.1221(4)$ & $0.43(1)$ & $216(3)$ & 2.96 \\
\hline
\end{tabular}

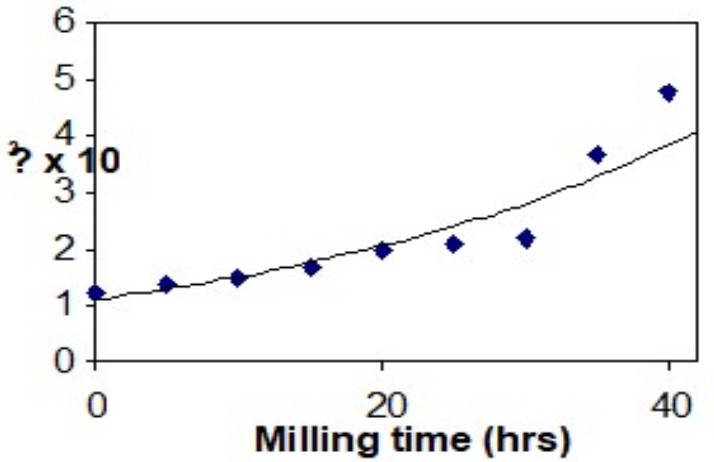

(a)

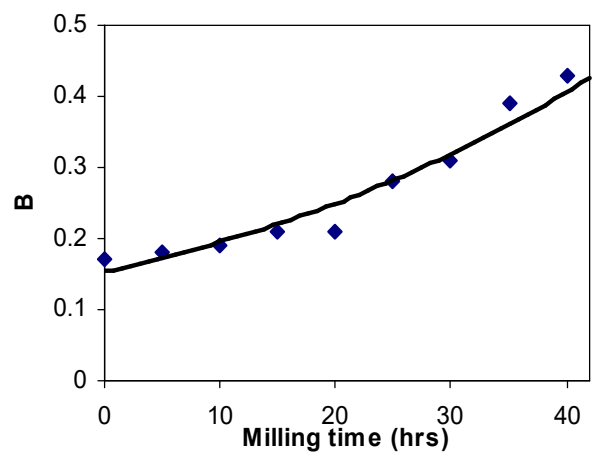

(c)

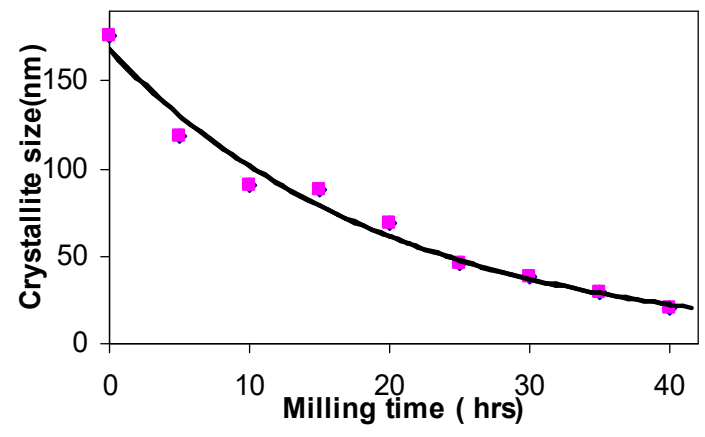

(b)

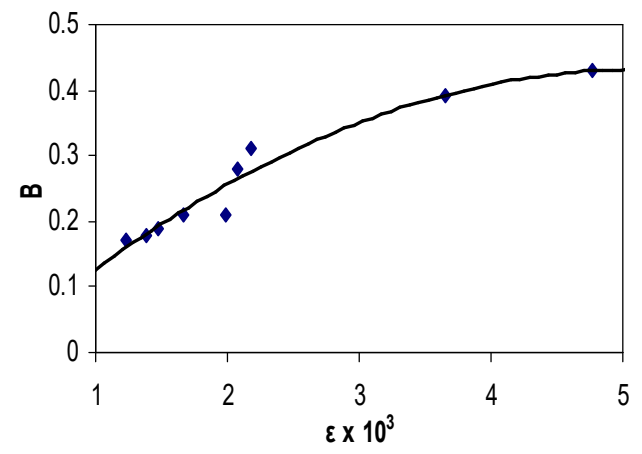

(d)

Fig.-3: Milling Time versus (a) Lattice Strain, (b) Crystallite Size and (c)Debye-Waller Factor and (d)Lattice Strain versus Debye-Waller Factor Curves for W.

Both lattice strain and Debye-Waller factor increase with milling time. This is similar to the observations of earlier workers $8,9,10$ and Gopi Krishna and Sirdeshmukh ${ }^{13}$. The Debye-Waller factor increases with milling time and lattice strain in a slightly non-linear fashion and crystallite size decreases with milling time. An extrapolation of the B versus $\varepsilon$ curve to $\varepsilon=0$ gives the values of Debye-Waller factor $0.12 \AA^{2}$. The zero-strain values of the Debye-Waller factor are less than the values for the initial samples. The zero strain Debye-Waller factors of $0.12 \AA^{2}$ for w is close to the value of $0.18 \AA^{2}$ obtained by earlier worker ${ }^{5}$ using powder X-ray diffraction. Thus, the Debye-Waller factors of w powder samples carry an effect due to lattice strain. While comparing the Debye-Waller factors calculated from the lattice dynamical models with experimental results, Vetelino et $a l^{21}$ have attributed the difference to inaccuracies in the experimental values caused by neglecting the TDS corrections. The repeated milling of the powder sample leads to lattice distortion which gives rise to microstrains in the lattice. These microstrains increase the contribution of the static component of the Debye-Waller factor. Thus both lattice strain and the observed Debye-Waller factor, which is the sum of static and thermal components, increase with milling time. Thus, whenever Debye-Waller factors are determined from X-ray intensities on powder samples, it is desirable to estimate the lattice strain and if the strain is large, a suitable correction is to be made as done in the present study.

Figures- 4 and 5 show SEM images of ball milled nano tungsten powders, after 0 , and 5 hours of highenergy planetary milling. The milled powders show an irregular morphology with avery fine particle 
RASĀYAN J. Chem.

Vol. 13 | No. 4 |2193-2199| October - December | 2020

size, approximately $50 \mathrm{~nm}$. Glyde ${ }^{22}$ derived the following relation between the energy of vacancy formation $\left(E_{f}\right)$ and the Debye temperature $(\theta)$ of a solid. The relation is-

$$
E_{\mathrm{f}}=\mathrm{A}(\mathrm{k} / \mathrm{h})^{2} \mathrm{M} \theta^{2} \mathrm{a}^{2}
$$

Where $\mathrm{a}$ is the interatomic spacing, $\mathrm{A}$ a constant shown to be equal to $1.17 \times 10^{-2}, \mathrm{M}$ the molecular weight and $h$ and $k$ are the Plank's and the Boltzmann's constants, respectively. Glyde recommended the use of $\mathrm{X}$-ray based values in eq. (15). The validity of eq.(15) was verified for severalfcc, bcc and hcp metals ${ }^{23}$ and Purushotham. ${ }^{24-29}$ Therefore, the X-ray Debye temperatures obtained in the present work have been used to study the variation of vacancy formation energy as a function of lattice strain in $\mathrm{W}$. The values of vacancy formation energies are also included in Table-1.

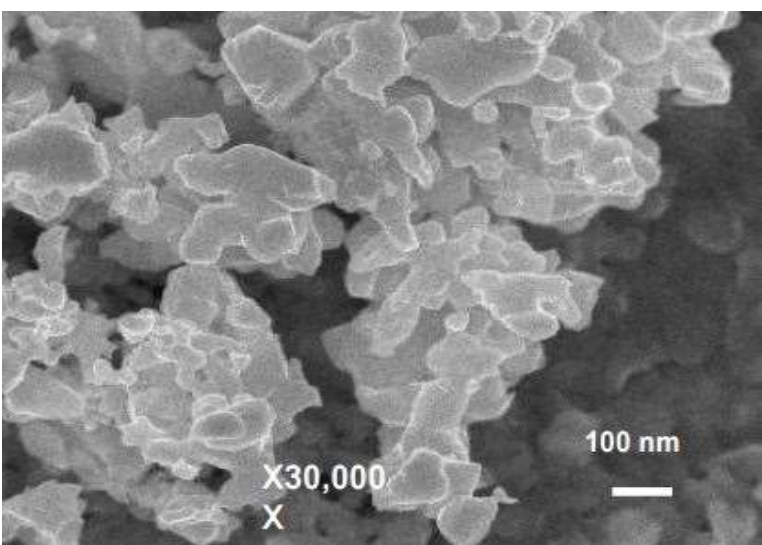

Fig.-4: SEM Image of the High Energy Planetary Ballmilled Nano-crystalline Tungsten Nanopowder Particle Size 500nm for Initial Sample (0 hours)

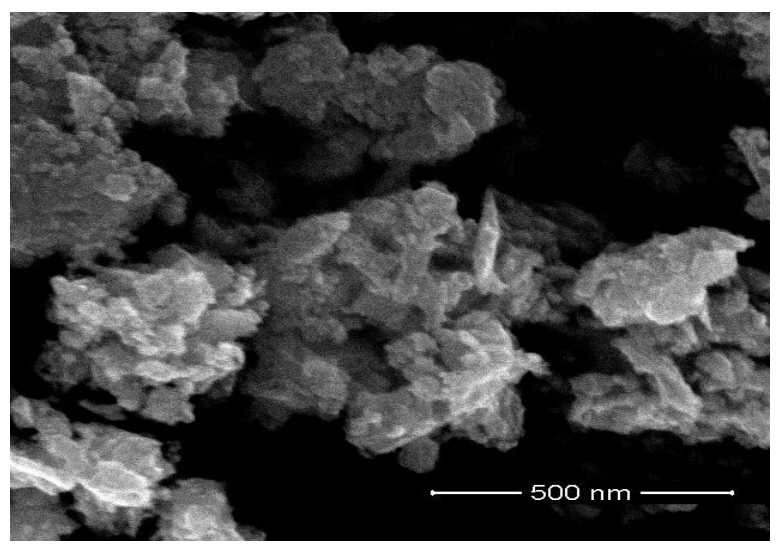

Fig.-5: SEM Image of the High Energy Planetary Ballmilled Nanocrystalline Tungsten Nanopowder Particle Size $100 \mathrm{~nm}$ for 5 hours

\section{CONCLUSION}

Tungsten powder was strained by milling for 40 hours. From a study of X-ray diffractograms recorded at different stages of milling, it is observed that milling for 40 hours has a systematic effect on the particle size. However, the milling produces lattice strain and also enhances the effective Debye-Waller factor. By extrapolation of the plot between the Debye-Waller factor and the lattice strain, the zero strain DebyeWaller factors are obtained for $\mathrm{W}$. The variation of energy of vacancy formation as a function of lattice strain has been studied.

\section{ACKNOWLEDGEMENT}

The author is grateful to Dr. K. Madangopal, AD, MG, Indigenous BARC Scanning Electron Microscope (SEM), Bhabha Atomic Research Centre,Trombay, Mumbai - 400 085, India for his help in the SEM investigation images. The author expresses his sincere thanks to Prof. D.B. Sirdeshmukh and Prof. N. Gopikrishna for useful suggestions. The author is grateful to the management of SR University, Ananthasagar, Warangal Urban, Telangana, India for permitting to use the Research Laboratory and undertake the reported investigations.

\section{REFERENCES}

1. V. Ashok Kumar, P. Sammaiah, Science Direct Materials Today: Proceedings, 5, 1(2017), DOI: 10.1016/j.matpr.2018.08.059

2. P. Srikanth Rao, D. Manoj Kumar, J. Gopikrishna, Science Direct Materials Today: Proceedings, 5(1), 1264 (2018).

3. Ch. Shiva Krishna, J. Manoj Kumar, International Journal of Materials Science,12(4), 627 (2017).

4. P. Satish Kumar, CH.S.R. Sastry, Ch. Devaraju, Materials Today Proceedings Elsevier,4(2), 330 (2017), DOI:10.1016/j.matpr.2017.01.029

5. E. Purushotham, Journal of Engineering Science and Technology Review, 7(1), 41 (2014).

6. E. Purushotham, Rasayan Journal of Chemistry, 12(4), 1676(2019), DOI: $10.31788 /$ RJC.2019.1245132 
RASĀYAN J. Chem.

Vol. 13 | No. 4 |2193-2199| October - December | 2020

7. E. Purushotham, N. Gopi Krishna, Indian Journal of Physics, 87(8), 982(2013), DOI: $10.1007 / \mathrm{s} 12648-013-0396-z$

8. E. Purushotham, N. Gopi Krishna, Bulletin of Materials Science, 36(6), 973(2013)

9. E. Purushotham, Chemistry and Materials Research, 7(2), 1 (2015)

10. D. B. Sirdeshmukh, K.G. Subhadra, K.A. Hussain, N. Gopi Krishna and B. Raghavendra Rao, Crystal Research Technology, 28, 15(1993), DOI:10.1002/crat.2170280104

11. N. Gopi Krishna and D.B. Sirdeshmukh, Indian Journal of Pure \& Applied Physics, 31, 198(1993)

12. D.R. Chipman and A. Paskin, Journal of Applied Physics, 30, 1938(1959).

13. H.P. Klug and L.E. Alexander, (1974), X-ray Diffraction Procedures (John Wiley and Sons, U.S.A.), DOI: $10.1002 /$ bbpc. 19750790622

14. D. T. Cromer, and J. T. Waber, Acta Crystllograica, 18, 104(1965), DOI: $10.1107 / \mathrm{S} 0365110 \mathrm{X} 6500018 \mathrm{X}$

15. International Tables for X-ray Crystallography (1968), Vol. III (Kynoch Press, Birmingham), DOI: $10.1107 / 97809553602060000577$

16. D.T. Cromer and D. Liberman, Journal of Chemistry and Physics, 53, 1891(1970), DOI: $10.1063 / 1.1674266$

17. G.C. Benson and E.K. Gill, (1966), Table of Integral Functions Related to Debye-Waller factor, National Research Council of Canada, Ottawa.

18. R. Bharati, P.B. Rehani,B. Joshi, N. Kirit and L. Arun Pratap, Indian Journal of Pure and Applied Physics, 44, 157(2006).

19. A.J.C. Wilson, (1949), X-ray Optics (Methuen, London).

20. E.F. Kaelble, Handbook of X-rays (New York Mc Graw ill) (1967)

21. J.F. Vetelino, S.P. Gaur, and S.S. Mitra, Physics Review B5, 2360 (1972).

22. H.R. Glyde, Journal of Physics and Chemistry Solids (G.B) 28, 2061(1967).

23. Micro-and Macro-Properties of Solids (Springer Series in Material Science)(2006)

24. E. Purushotham, American Journal of Nanosciences, 7, 23(2019), DOI:10.11648/j.ajn.20190503.11

25. E. Purushotham, American Journal of Quantum Chemistry and Molecular Spectroscopy, 3,37(2019), DOI: 10.11648/j.ajqcms.20190302.12

26. E. Purushotham, International Journal of Fluid Mechanics \& Thermal Sciences, 5(3), 63 (2019), DOI:10.11648/j.ijfmts.20190503.11

27. E. Purushotham E, International Journal of Chemistry Studies, 3(2), 28(2019)

28. E. Purushotham, Modern Chemistry, 7(1), 5(2018), DOI:10.11648/j.mc.20190701.12

29. E. Purushotham, International Journal of High Energy Physics, 5(1), 1(2018), DOI: 10.11648/j.ijhep.20180501.11

[RJC-5788/2020] 